\title{
In-House: An Ensemble of Pre-Existing Off-the-Shelf Parsers
}

\author{
Yusuke Miyao*, Stephan Oepen ${ }^{\curvearrowright}$, and Daniel Zeman $\diamond$ \\ * National Institute of Informatics, Tokyo \\ - University of Oslo, Department of Informatics \\ ${ }^{\circ}$ Potsdam University, Department of Linguistics \\ $\diamond$ Charles University in Prague, Faculty of Mathematics and Physics, Institute of Formal and Applied Linguistics \\ yusuke@nit.ac.jp, oe@ifi.uio.no, zeman@ufal.mff.cuni.cz
}

\begin{abstract}
This submission to the open track of Task 8 at SemEval 2014 seeks to connect the Task to pre-existing, 'in-house' parsing systems for the same types of target semantic dependency graphs.
\end{abstract}

\section{Background and Motivation}

The three target representations for Task 8 at SemEval 2014, Broad-Coverage Semantic Dependency Parsing (SDP; Oepen et al., 2014), are rooted in language engineering efforts that have been under continuous development for at least the past decade. The gold-standard semantic dependency graphs used for training and testing in the Task result from largely manual annotation, in part re-purposing and adapting resources like the Penn Treebank (PTB; Marcus et al., 1993), PropBank (Palmer et al., 2005), and others. But the groups who prepared the SDP target data have also worked in parallel on automated parsing systems for these representations.

Thus, for each of the target representations, there is a pre-existing parser, often developed in parallel to the creation of the target dependency graphs, viz. (a) for the DM representation, the parser of the hand-engineered LinGO English Resource Grammar (ERG; Flickinger, 2000); (b) for PAS, the Enju parsing system (Miyao, 2006), with its probabilistic HPSG acquired through linguistic projection of the PTB; and (c) for PCEDT, the scenario for English analysis within the Treex framework (Popel and Žabokrtský, 2010), combining data-driven dependency parsing with handengineered tectogrammatical conversion. At least

This work is licenced under a Creative Commons Attribution 4.0 International License; page numbers and the proceedings footer are added by the organizers. http:// creativecommons.org/licenses/by/4.0/ for DM and PAS, these parsers have been extensively engineered and applied successfully in a variety of applications, hence represent relevant points of comparison. Through this 'in-house' submission (of our 'own' parsers to our 'own' task), we hope to facilitate the comparison of different approaches submitted to the Task with this pre-existing line of parser engineering.

\section{DM: The English Resource Grammar}

Semantic dependency graphs in the DM target representation, DELPH-IN MRS-Derived Bi-Lexical Dependencies, stem from a two-step 'reduction' (simplification) of the underspecified logicalform meaning representations output natively by the ERG parser, which implements the linguistic framework of Head-Driven Phrase Structure Grammar (HPSG; Pollard and Sag, 1994). Goldstandard DM training and test data for the Task were derived from the manually annotated DeepBank Treebank (Flickinger et al., 2012), which pairs Sections 00-21 of the venerable PTB Wall Street Journal (WSJ) Corpus with complete ERGcompatible HPSG syntactico-semantic analyses. DeepBank as well as the ERG rely on Minimal Recursion Semantics (MRS; Copestake et al., 2005) for meaning representation, such that the exact same post-processing steps could be applied to the parser outputs as were used in originally reducing the gold-standard MRSs from DeepBank into the SDP bi-lexical semantic dependency graphs.

Parsing Setup The ERG parsing system is a hybrid, combining (a) the hand-built, broad-coverage ERG with (b) an efficient chart parser for unification grammars and (c) a conditional probability distribution over candidate analyses. The parser most commonly used with the ERG, called PET (Callmeier, 2002), ${ }^{1}$ constructs a complete,

\footnotetext{
${ }^{1}$ The SDP test data was parsed using the 1212 release of the ERG, using PET and converter versions from what
} 
subsumption-based parse forest of partial HPSG derivations (Oepen and Carroll, 2000), and then extracts from the forest $n$-best lists (in globally correct rank order) of complete analyses according to a discriminative parse ranking model (Zhang et al., 2007). For our experiments, we trained the parse ranker on Sections 00-20 of DeepBank and otherwise used the default, non-pruning development configuration, which is optimized for accuracy. In this setup, ERG parsing on average takes close to ten seconds per sentence.

Post-Parsing Conversion After parsing, MRSs are reduced to DM bi-lexical semantic dependencies in two steps. First, Oepen and Lønning (2006) define a conversion to variable-free Elementary Dependency Structures (EDS), which (a) maps each predication in the MRS logical-form meaning representation to a node in a dependency graph and (b) transforms argument relations represented by shared logical variables into directed dependency links between graph nodes. This first step of the conversion is 'mildly' lossy, in that some scope-related information is discarded; the EDS graph, however, will contain the same number of nodes and the same set of argument dependencies as there are predications and semantic role assignments in the original MRS. In particular, the EDS may still reflect non-lexical semantic predications introduced by grammatical constructions like covert quantifiers, nominalization, compounding, or implicit conjunction. ${ }^{2}$

Second, in another conversion step that is not information-preserving, the EDS graphs are further reduced into strictly bi-lexical form, i.e. a set of directed, binary dependency relations holding exclusively between lexical units. This conversion is defined by Ivanova et al. (2012) and seeks to (a) project some aspects of construction semantics onto word-to-word dependencies (for example introducing specific dependency types for compounding or implicit conjunction) and (b) relate the linguistically informed ERG-internal tokenization to the conventions of the PTB. ${ }^{3}$ Seeing as both

is called the LOGON SVN trunk as of January 2014; see http: //moin.delph-in. net/LogonTop for detail.

${ }^{2}$ Conversely, semantically vacuous parts of the original input (e.g. infinitival particles, complementizers, relative pronouns, argument-marking prepositions, auxiliaries, and most punctuation marks) were not represented in the MRS in the first place, hence have no bearing on the conversion.

${ }^{3}$ Adaptations of tokenization encompass splitting 'multiword' ERG tokens (like such as or ad hoc), as well as 'hiding' ERG token boundaries at hyphens or slashes (e.g. 77-year- conversion steps are by design lossy, DM semantic dependency graphs present a true subset of the information encoded in the full, original MRS.

\section{PAS: The Enju Parsing System}

Enju Predicate-Argument Structures (PAS) are derived from the automatic HPSG-style annotation of the PTB, which was primarily used for the development of the Enju parsing system ${ }^{4}$ (Miyao, 2006). A notable feature of this parser is that the grammar is not developed by hand; instead, the Enju HPSG-style treebank is first developed, and the grammar (or, more precisely, the vast majority of lexical entries) is automatically extracted from the treebank (Miyao et al., 2004). In this 'projection' step, PTB annotations such as empty categories and coindexation are used for deriving the semantic representations that correspond to HPSG derivations. Its probabilistic model for disambiguation is also trained using this treebank (Miyao and Tsujii, 2008). ${ }^{5}$

The PAS data set is an extraction of predicateargument structures from the Enju HPSG treebank. The Enju parser outputs results in 'readyto-use' formats like phrase structure trees and predicate-argument structures, as full HPSG analyses are not friendly to users who are not familiar with the HPSG theory. The gold-standard PAS target data in the Task was developed using this function; the conversion program from full HPSG analyses to predicate-argument structures was applied to the Enju Treebank.

Predicate-argument structures (PAS) represent word-to-word semantic dependencies, such as semantic subject and object. Each dependency type is represented with two elements: the type of the predicate, such as verb and adjective, and the argument label, such as ARG1 and ARG2. ${ }^{6}$

\footnotetext{
old), which the PTB does not split.

${ }^{4}$ See http://kmcs.nii.ac.jp/enju/.

${ }^{5}$ Abstractly similar to the ERG, the annotations of the Enju treebank instantiate the linguistic theory of HPSG. However, the two resources have been developed independently and implementation details are quite different. The most significant difference is that the Enju HPSG treebank is developed by linguistic projection of PTB annotations, and the Enju parser derived from the treebank; conversely, the ERG was predominantly manually crafted, and it was later applied in the DeepBank re-annotation of the WSJ Corpus.

${ }^{6}$ Full details of the predicate-argument structures in the Enju HPSG Treebank, are available in two documents linked from the Enju web site (see above), viz. the Enju Output Specification Manual and the XML Format Documentation.
} 
Parsing Setup Basically we used the publicly available package of the Enju parser 'as is' (see the above web site). We did not change default parsing parameters (beam width, etc.) and features. However, the release version of the Enju parser is trained with the HPSG treebank corresponding to the Penn Treebank WSJ Sections 2-21, which includes the test set of the Task (Section 21). Therefore, we re-trained the Enju parser using Sections $0-20$, and used this re-trained parser in preparing the PAS semantic dependency graphs in this ensemble submission.

Post-Parsing Conversion The dependency format of the Enju parser is almost equivalent to what is provided as the PAS data set in this shared task. Therefore, the post-parsing conversion for the PAS data involves only formatting, viz. (a) format conversion into the tabular file format of the Task; and (b) insertion of dummy relations for punctuation tokens ignored in the output of Enju. ${ }^{7}$

\section{PCEDT: The Treex Parsing Scenario}

The Prague Czech-English Dependency Treebank (PCEDT; Hajič et al., 2012) ${ }^{8}$ is a set of parallel dependency trees over the same WSJ texts from the Penn Treebank, and their Czech translations. Similarly to other treebanks in the Prague family, there are two layers of syntactic annotation: analytical (a-trees) and tectogrammatical (t-trees). Unlike for the other two representations used in the Task, for PCEDT there is no pre-existing parsing system designed to deliver the full scale of annotations of the SDP gold-standard data. The closest available match is a parsing scenario implemented in the Treex natural language processing framework.

Parsing Setup Treex ${ }^{9}$ (Popel and Žabokrtský, 2010) is a modular, open-source framework originally developed for transfer-based machine translation. It can accomplish any NLP-related task by sequentially applying to the same piece of data various blocks of code. Blocks operate on a common data structure and are chained in scenarios.

Some early experiments with scenarios for tectogrammatical analysis of English were described by Klimeš (2007). It is of interest that they report

\footnotetext{
${ }^{7}$ The Enju parser ignores tokens tagged as ‘.', while the PAS representation includes them with dummy relations; thus, missing periods are inserted in post-processing by comparison to the original PTB token sequence.

${ }^{8}$ See http://ufal.mff.cuni.cz/pcedt2.0/.

${ }^{9}$ See http://ufal.mff.cuni.cz/treex/.
}

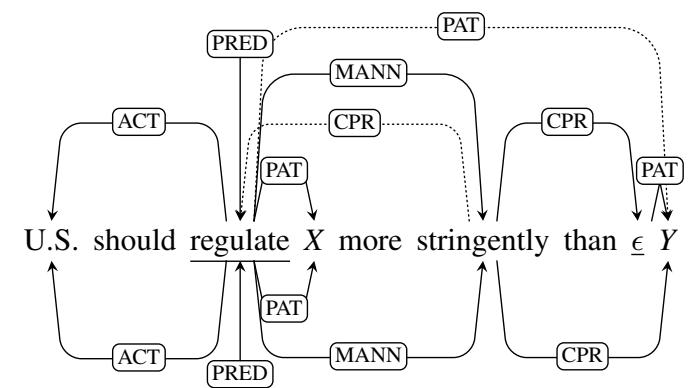

Figure 1: PCEDT asserts two copies of the token regulate (shown here as 'regulate' and ' $\epsilon$ ', underlined). Projecting t-nodes onto the original tokens, required by the SDP data format, means that the $\epsilon$ node will be merged with regulate. The edges going to and from $\epsilon$ will now lead to and from regulate (see the dotted arcs), which results in a cycle. To get rid of the cycle, we skip $\epsilon$ and connect directly its children, as shown in the final SDP graph below the sentence.

an $\mathrm{F}_{1}$ score of assigning functors (dependency labels in PCEDT terminology) of $70.3 \%$; however, their results are not directly comparable to ours.

Due to the modular nature of Treex, there are various conceivable scenarios to get the t-tree of a sentence. We use the default scenario that consists of 48 blocks: two initial blocks (reading the input), one final block (writing the output), two $\mathrm{A} 2 \mathrm{~N}$ blocks (named entity recognition), twelve W2A blocks (dependency parsing at the analytical layer) and $31 \mathrm{~A} 2 \mathrm{~T}$ and $\mathrm{T} 2 \mathrm{~T}$ blocks (creating the $\mathrm{t}$-tree based on the a-tree).

Most blocks are highly specialized in one particular subtask (e.g. there is a block just to make sure that quotation marks are attached to the root of the quoted subtree). A few blocks are responsible for the bulk of the work. The a-tree is constructed by a block that contains the MST Parser (McDonald et al., 2005), trained on the CoNLL 2007 English data (Nivre et al., 2007), i.e. Sections 2-11 of the PTB, converted to dependencies. The annotation style of CoNLL 2007 differs from PCEDT 2.0, and thus the unlabeled attachment score of the analytical parser is only $66 \%$.

Obviously one could expect better results if we retrained the MST Parser directly on the PCEDT a-trees, and on the whole training data. The only reason why we did not do so was lack of time. Our results thus really demonstrate what is available 'off-the-shelf'; on the other hand, the PCEDT component of our ensemble fails to set any 'upper bound' of output quality, as it definitely is not bet- 


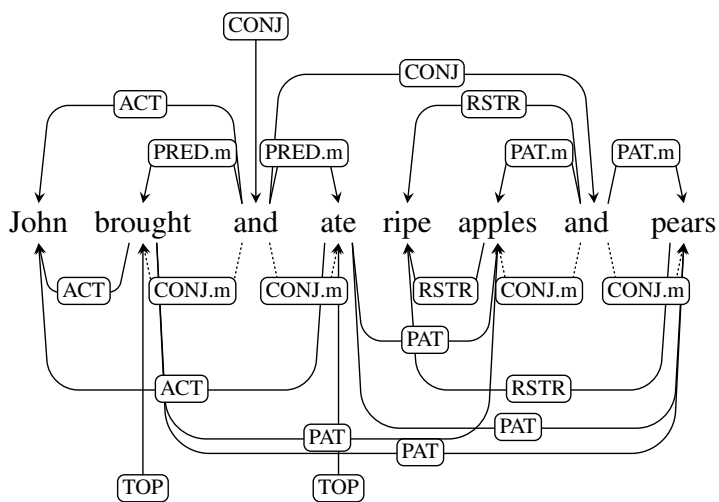

Figure 2: Coordination in PCEDT t-tree (above) and in the corresponding SDP graph (below).

ter informed than the other systems participating in the Task.

Functor assignment is done heuristically, based on POS tags and function words. The primary focus of the scenario was on functors that could help machine translation, thus it only generated 25 different labels (of the total set of 65 labels in the SDP gold-standard data) ${ }^{10}$ and left about $12 \%$ of all nodes without functors. Precision peaks at $78 \%$ for $\mathrm{ACT}$ (or) relations, while the most frequent error type (besides labelless dependencies) is a falsely proposed RSTR(iction) relation. Both ACT and RSTR are among the most frequent dependency types in PCEDT.

Post-Parsing Conversion Once the t-tree has been constructed, it is converted to the PCEDT target representation of the Task, using the same conversion code that was used to prepare the goldstandard SDP data. ${ }^{11}$

SDP graphs are defined over surface tokens but the set of nodes of a t-tree need not correspond one-to-one to the set of tokens. For example, there are no t-nodes for punctuation and function words (except in coordination); these tokens are rendered as semantically vacuous in SDP, i.e. they do not participate in edges. On the other hand, t-trees can contain generated nodes, which represent elided words and do not correspond to any surface to-

\footnotetext{
${ }^{10}$ The system was able to output the following functors (ordered in the descending order of their frequency in the system output): RSTR, PAT, ACT, CONJ.member, APP, MANN, LOC, TWHEN, DISJ.member, BEN, RHEM, PREC, ACMP, MEANS, ADVS.member, CPR, EXT, DIR3, CAUS, COND, TSIN, REG, DIR2, CNCS, and TTILL.

${ }^{11}$ In the SDP context, the target representation derived from the PCEDT is called by the same name as the original treebank; but note that the PCEDT semantic dependency graphs only encode a subset of the information annotated at the tectogrammatical layer of the full treebank.
}

\begin{tabular}{l|cc|cc|cc} 
& \multicolumn{2}{|c|}{ DM } & \multicolumn{2}{c|}{ PAS } & \multicolumn{2}{c}{ PCEDT } \\
& LF & LM & LF & LM & LF & LM \\
\hline Priberam & .8916 & .2685 & .9176 & .3783 & .7790 & .1068 \\
In-House & .9246 & .4807 & .9206 & .4384 & .4315 & .0030 \\
& UF & UM & UF & UM & UF & UM \\
\hline Priberam & .9032 & .2990 & .9281 & .3924 & .8903 & .3071 \\
In-House & .9349 & .5230 & .9317 & .4429 & .6919 & .0148
\end{tabular}

Table 1: End-to-end 'in-house' parsing results.

ken. Most generated nodes are leaves and, thus, can simply be omitted from the SDP graphs. Other generated nodes are copies of normal nodes and they are linked to the same token to which the source node is mapped. As a result, one token can appear at several different positions in the tree; if we project these occurrences into one node, the graph will contain cycles. We decided to remove all generated nodes causing cycles. Their children are attached to their parents and inherit the functor of the generated node (Figure 1). The conversion procedure also removes cycles caused by more fine-grained tokenization of the t-layer.

Furthermore, t-trees use technical edges to capture paratactic constructions where the relations are not 'true' dependencies. The conversion procedure extracts true dependency relations: Each conjunct is linked to the parent or to a shared child of the coordination. In addition, there are also links from the conjunction to the conjuncts and they are labeled CONJ.m(ember). These links preserve the paratactic structure (which can even be nested) and the type of coordination. See Figure 2 for an example.

\section{Results and Reflections}

Seeing as our 'in-house' parsers are not directly trained on the semantic dependency graphs provided for the Task, but rather are built from additional linguistic resources, we submitted results from the parsing pipelines sketched in Sections 2 to 4 above to the open SDP track. Table 1 summarizes parser performance in terms of labeled and unlabeled $F_{1}$ (LF and UF) ${ }^{12}$ and fullsentence exact match (LM and UM), comparing to the best-performing submission (dubbed Priberam; Martins and Almeida, 2014) to this track. Judging by the official SDP evaluation metric, average labeled $F_{1}$ over the three representations, our ensemble ranked last among six participating

\footnotetext{
${ }^{12}$ Our ensemble members exhibit comparatively small differences in recall vs. precision.
} 
teams; in terms of unlabeled average $F_{1}$, the "inhouse' submission achieved the fourth rank.

As explained in the task description (Oepen et al., 2014), parts of the WSJ Corpus were excluded from the SDP training and testing data because of gaps in the DeepBank and Enju treebanks, and to exclude cyclic dependency graphs, which can sometimes arise in the DM and PCEDT conversions. For these reasons, one has to allow for the possibility that the testing data is positively biased towards our ensemble members. ${ }^{13}$ But even with this caveat, it seems fair to observe that the ERG and Enju parsers both are very competitive for the DM and PAS target representations, respectively, specifically so when judged in exact match scores. A possible explanation for these results lies in the depth of grammatical information available to these parsers, where DM or PAS semantic dependency graphs are merely a simpliefied view on the complete underlying HPSG analyses. These parsers have performed well in earlier contrastive evaluation too (Miyao et al., 2007; Bender et al., 2011; Ivanova et al., 2013; inter alios).

Results for the Treex English parsing scenario, on the other hand, show that this ensemble member is not fine-tuned for the PCEDT target representation; due to the reasons mentioned above, its performance even falls behind the shared task baseline. As is evident from the comparison of labeled vs. unlabeled $\mathrm{F}_{1}$ scores, (a) the PCEDT parser is comparatively stronger at recovering semantic dependency structure than at assigning labels, and (b) about the same appears to be the case for the best-performing Priberam system (on this target representation).

\section{Acknowledgements}

Data preparation and large-scale parsing in the DM target representation was supported through access to the ABEL high-performance computing facilities at the University of Oslo, and we acknowledge the Scientific Computing staff at UiO, the Norwegian Metacenter for Computational Science, and the Norwegian tax payers. This project has been supported by the infrastructural funding

\footnotetext{
${ }^{13}$ There is no specific evidence that the WSJ sentences excluded in the Task for technical issues in either of the underlying treebanks or conversion procedures would be comparatively much easier to parse for other submissions than for the members of our 'in-house' ensemble, but unlike other systems these parsers 'had a vote' in the selection of the data, particularly so for the DM and PAS target representations.
}

by the Ministry of Education, Youth and Sports of the Czech Republic (CEP ID LM2010013).

\section{References}

Bender, E. M., Flickinger, D., Oepen, S., and Zhang, Y. (2011). Parser evaluation over local and non-local deep dependencies in a large corpus. In Proceedings of the 2011 Conference on Empirical Methods in Natural Language Processing (p. 397-408). Edinburgh, Scotland, UK.

Callmeier, U. (2002). Preprocessing and encoding techniques in PET. In S. Oepen, D. Flickinger, J. Tsujii, and H. Uszkoreit (Eds.), Collaborative language engineering. A case study in effcient grammar-based processing (p. $127-140$ ). Stanford, CA: CSLI Publications.

Copestake, A., Flickinger, D., Pollard, C., and Sag, I. A. (2005). Minimal Recursion Semantics. An introduction. Research on Language and Computation, 3(4), 281-332.

Flickinger, D. (2000). On building a more efficient grammar by exploiting types. Natural Language Engineering, 6 (1), 15-28.

Flickinger, D., Zhang, Y., and Kordoni, V. (2012). DeepBank. A dynamically annotated treebank of the Wall Street Journal. In Proceedings of the 11th International Workshop on Treebanks and Linguistic Theories (p. 85-96). Lisbon, Portugal: Edições Colibri.

Hajič, J., Hajičová, E., Panevová, J., Sgall, P., Bojar, O., Cinková, S., ... Žabokrtský, Z. (2012). Announcing Prague Czech-English Dependency Treebank 2.0. In Proceedings of the 8th International Conference on Language Resources and Evaluation (p. 3153 -3160). Istanbul, Turkey.

Ivanova, A., Oepen, S., Dridan, R., Flickinger, D., and Øvrelid, L. (2013). On different approaches to syntactic analysis into bi-lexical dependencies. An empirical comparison of direct, PCFGbased, and HPSG-based parsers. In Proceedings of the 13th International Conference on Parsing Technologies (p. 63 -72). Nara, Japan.

Ivanova, A., Oepen, S., Øvrelid, L., and Flickinger, D. (2012). Who did what to whom? 
A contrastive study of syntacto-semantic dependencies. In Proceedings of the Sixth Linguistic Annotation Workshop (p. 2-11). Jeju, Republic of Korea.

Klimeš, V. (2007). Transformation-based tectogrammatical dependency analysis of English. In V. Matoušek and P. Mautner (Eds.), Text, speech and dialogue 2007, LNAI 4629 (p. $15-$ 22). Berlin / Heidelberg, Germany: Springer.

Marcus, M., Santorini, B., and Marcinkiewicz, M. A. (1993). Building a large annotated corpora of English: The Penn Treebank. Computational Linguistics, 19, 313-330.

Martins, A. F. T., and Almeida, M. S. C. (2014). Priberam. A turbo semantic parser with second order features. In Proceedings of the 8th International Workshop on Semantic Evaluation. Dublin, Ireland.

McDonald, R., Pereira, F., Ribarov, K., and Hajič, J. (2005). Non-projective dependency parsing using spanning tree algorithms. In Proceedings of the Human Language Technology Conference and Conference on Empirical Methods in Natural Language Processing (p. 523-530). Vancouver, British Columbia, Canada.

Miyao, Y. (2006). From linguistic theory to syntactic analysis. Corpus-oriented grammar development and feature forest model. Doctoral Dissertation, University of Tokyo, Tokyo, Japan.

Miyao, Y., Ninomiya, T., and Tsujii, J. (2004). Corpus-oriented grammar development for acquiring a Head-Driven Phrase Structure Grammar from the Penn Treebank. In Proceedings of the 1st International Joint Conference on Natural Language Processing (p. 684-693).

Miyao, Y., Sagae, K., and Tsujii, J. (2007). Towards framework-independent evaluation of deep linguistic parsers. In Proceedings of the 2007 Workshop on Grammar Engineering across Frameworks (p. 238-258). Palo Alto, California.

Miyao, Y., and Tsujii, J. (2008). Feature forest models for probabilistic HPSG parsing. Computational Linguistics, 34(1), 35-80.
Nivre, J., Hall, J., Kübler, S., McDonald, R., Nilsson, J., Riedel, S., and Yuret, D. (2007). The CoNLL 2007 shared task on dependency parsing. In Proceedings of the 2007 Joint Conference on Empirical Methods in Natural Language Processing and Conference on Natural Language Learning (p. 915-932). Prague, Czech Republic.

Oepen, S., and Carroll, J. (2000). Ambiguity packing in constraint-based parsing. Practical results. In Proceedings of the 1st Meeting of the North American Chapter of the Association for Computational Linguistics (p. 162 -169). Seattle, WA, USA.

Oepen, S., Kuhlmann, M., Miyao, Y., Zeman, D., Flickinger, D., Hajič, J., ... Zhang, Y. (2014). SemEval 2014 Task 8. Broad-coverage semantic dependency parsing. In Proceedings of the 8th International Workshop on Semantic Evaluation. Dublin, Ireland.

Oepen, S., and Lønning, J. T. (2006). Discriminant-based MRS banking. In Proceedings of the 5th International Conference on Language Resources and Evaluation (p. 12501255). Genoa, Italy.

Palmer, M., Gildea, D., and Kingsbury, P. (2005). The Proposition Bank. A corpus annotated with semantic roles. Computational Linguistics, 31(1), $71-106$.

Pollard, C., and Sag, I. A. (1994). Head-Driven Phrase Structure Grammar. Chicago, USA: The University of Chicago Press.

Popel, M., and Žabokrtský, Z. (2010). TectoMT. Modular NLP framework. Advances in Natural Language Processing, 293-304.

Zhang, Y., Oepen, S., and Carroll, J. (2007). Efficiency in unification-based n-best parsing. In Proceedings of the 10th International Conference on Parsing Technologies (p. 48-59). Prague, Czech Republic. 